\title{
The nosological position of concentric lacunar leucoencephalopathy
}

\author{
S. CURRIE, ${ }^{1}$ A. H. ROBERTS, AND H. URICH \\ The London Hospital, Whitechapel, London
}

In 1960 Grcević reported an apparently unique case. The patient, a woman, had been well until the age of 30. In the following three years she became 'almost blind', her vision deteriorating in an illness characterized by exacerbations and partial remissions. She then became progressively demented, developing abruptly a hemiparesis and aphasia six months before her death in epileptic status at the age of 35 . At necropsy extensive changes were found in the white matter of the rostral two-thirds of the cerebral hemispheres consisting of concentric lacunae separated by glial septa. In the affected areas there was total loss of axons and myelin sheaths. The lesions bore a superficial similarity to the concentric sclerosis of Baló (1928) from which they differed in being destructive and not demyelinating. The author discussed the nosological position of the condition which he named 'concentric lacunar leucoencephalopathy', and its relationship to demyelinating diseases, without reaching definite conclusions.

We report a case in which the association of similar lesions in the white matter with patchy demyelination in the optic pathways and an acute necrotizing myelopathy suggested a close relationship to the demyelinating diseases, particularly to neuromyelitis optica.

\section{CASE REPORT}

CLINICAL HISTORY Mr. G.R., a labourer, was admitted to his local hospital at the age of 64 in August 1966 with painless deterioration in the vision of both eyes. This appeared to have developed suddenly overnight. When he was seen two weeks later a left homonymous field defect was found which over the next two weeks progressed to complete blindness. His memory had become defective during the same period. Vision in the left eye was said to have been poor for years, but three years earlier severe myopia had apparently been corrected by an optician. Two weeks after the sudden deterioration in vision lumbar puncture was performed; the cerebrospinal

'Present address: Newcastle General Hospital, Newcastle upon Tyne, 4. fluid was found to contain $208 \mathrm{mg} . / 100 \mathrm{ml}$. protein but no other abnormality, and manometrics were normal. The Wassermann reaction was negative in blood and fluid. It was thought that the patient might have a suprasellar lesion and he was transferred to a neurosurgical centre. There he was found to be disorientated. The eyes were myopic and the optic discs were noted to be pale, but there appeared to be normal pupillary reactions despite imperception of light. There was a mild impairment of superficial sensory perception over the left side of the body. At ventriculography the fluid was xanthochromic and turbid, containing 375 red blood cells and 4 white cells/cu. $\mathrm{mm}$ and $140 \mathrm{mg} / 100 \mathrm{ml}$. of protein; manometrics and radiology were normal. A right vertebral angiogram was normal. Infarction of both occipital lobes was considered the most probable diagnosis.

The patient's condition then remained unchanged until five months later in January 1967 he developed a sudden left hemiplegia from which he recovered within 24 hours. Three months after this a grand mal epileptic attack occurred, followed by transient left-sided weakness. He was admitted to a local hospital again. There he was found to be orientated and co-operative, but blind without light perception, the pupils reacting only sluggishly to light. The optic discs were noted to be pale. No disturbance of left-sided motor or sensory function was apparent. He was normotensive. An ESR and blood count were normal. A right carotid angiogram demonstrated atheroma at the origin of the internal carotid artery but no abnormality of the intracerebral vessels. The patient was treated with phenobarbitone and had only one further major seizure during the following year.

He then remained well again until October 1967, five weeks before his death, when a sudden episode of weakness of both legs occurred lasting half an hour. This recurred within a fortnight, again transiently, on this occasion after a long walk. Two days later he awoke in the night with severe lumbar pain and on the following morning was incontinent of urine and unable to move his legs. On admission to hospital a dense flaccid paraplegia was found with cutaneous sensory loss from the fourth thoracic dermatome caudally; bladder drainage was required.

He was transferred to the London Hospital (no. 369575) the next day. On admission he was apyrexial, but drowsy, disorientated, and euphoric. He was blind without light perception in either eye. Pupillary reactions were present 
but not in response to light, and were thought to indicate reflex attempts at convergence. The left eye was highly myopic and both optic discs were atrophic. The retinae and vessels were normal. Neck stiffness was present. Initially a flaccid paraplegia with slight weakness of the wrist extensors on the left was noted. The tendon reflexes were sluggish in the arms, with the exception of the triceps, which were brisk, and absent in the legs. There was little response to plantar stimulation. Respiration was largely diaphragmatic, only the upper intercostals contracting. There was anaesthesia from the second thoracic dermatome caudally. Later the same day the sensory level was found to have risen to the fourth cervical dermatome on the left and to the eighth on the right, and breathing had become entirely diaphragmatic. Routine investigations including peripheral blood examination and radiographs of the chest and spine were normal. The ESR was $20 \mathrm{~mm}$ in one hour. The cerebrospinal fluid contained 355 white cells/cu. mm, predominantly neutrophils, $40 \mathrm{mg} / 100 \mathrm{ml}$. protein, and 90 $/ 100 \mathrm{ml}$. sugar. The Wassermann and Lange colloidal gold reactions in the fluid were negative and no organisms were seen or cultured. A myelogram was normal. A presumptive diagnosis of infarction of the cord was made. Within two days the sensory level had risen to the second cervical dermatome, the arms had become asymmetrically weak with slight power remaining only proximally in deltoids and biceps, while the tendon reflexes could no longer be elicited. He continued to deteriorate, the arms becoming flaccid and totally paralysed the day before his death. He died on 6 November 1967, 11 days after the onset of persistent leg weakness.

NECROPSY FINDINGS (PM 354/67) The following features were observed: concentric lacunar leucoencephalopathy; retrobulbar neuritis; acute myelomalacia; deep femoral vein thrombosis; pulmonary embolism; and acute haemorrhagic cystitis.
EXAMINATION OF THE NERVOUS SYSTEM Macroscopic The brain $(1,176 \mathrm{~g})$ was externally normal. Coronal sections of the cerebral hemispheres revealed extensive lacunar softening in the white matter, involving both occipital lobes, parts of the right parietal and right temporal lobes, and the splenium of the corpus callosum (Fig. 1). The lacunae were arranged in approximately parallel, concentric rows, separated by lamellae of firm, white glial tissue. In the lateral parts of the white matter the lacunae were larger and more widely spaced out than those situated more medially. The cortex over the affected areas appeared normal. Right Ammon's horn was shrunken and discoloured. Both lateral geniculate bodies were atrophic. The intracranial portions of the optic nerves and the optic chiasm showed no obvious lesions. The ventricles were normal. No lesions were seen in the basal ganglia, brain-stem or cerebellum.

The spinal cord externally appeared swollen and discoloured from the lower cervical to the lower thoracic segments. Transverse sections showed central softening with partial peripheral preservation from $\mathrm{C} 4$ to $\mathrm{C}$. From C8 to T11 the cord appeared completely necrotic with patches of haemorrhagic discolouration. The lowest thoracic and all lumbar segments were fairly well preserved and showed central small patches of brownish discolouration. The anterior and posterior roots and the posterior root ganglia appeared normal.

Microscopic The cerebral cortex showed good preservation of its cyto-architecture with the exception of right Ammon's horn which showed a large defect in Sommer's sector of the pyramidal layer. Sections stained for myelin showed partial demyelination of the cortex in areas adjacent to the lesions in the white matter. This was most striking in the calcarine fissure where only the tips of the cuneus and the lingual gyrus remained fully myelinated, while in the deeper part of the fissure there was total loss of myelin both in the radial fibres and in

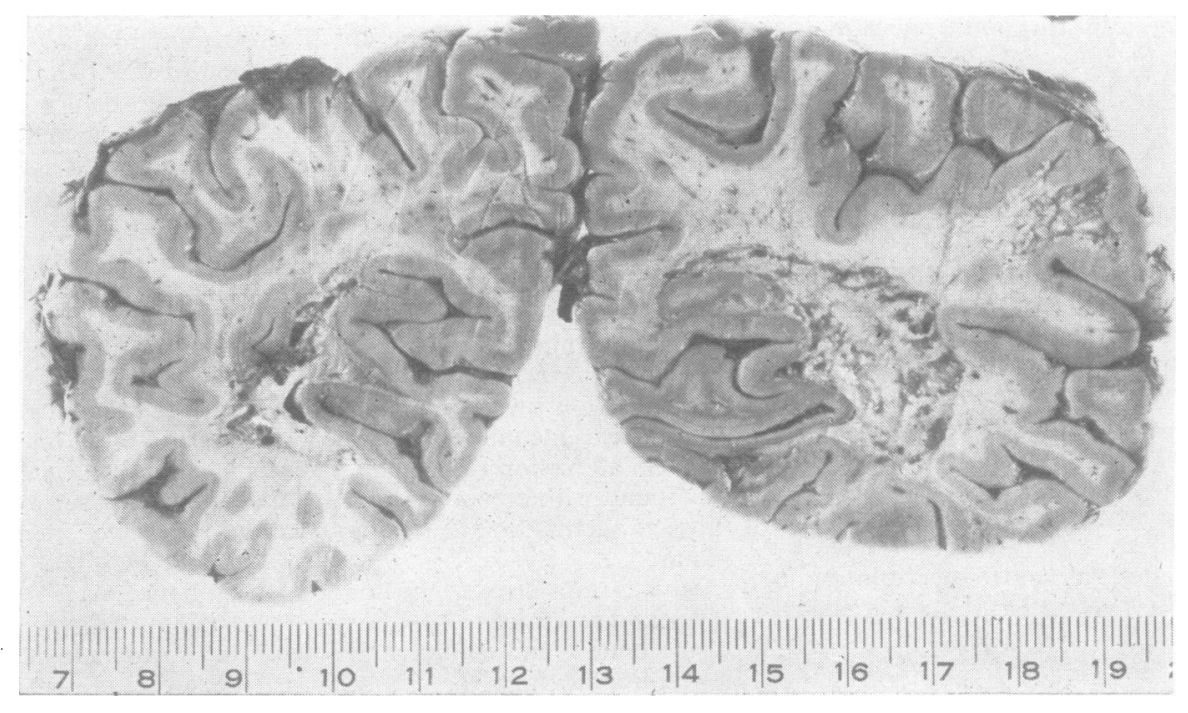

FIG. 1. Coronal section through both occipital lobes showing concentric arrangement of rows of lacunae, particularly on the right. 
the stria of Gennari (Fig. 2). There was a mild marginal gliosis throughout the occipital cortex and in addition a striking proliferation of large spider astrocytes in layer VI of the visual cortex in the floor of the calcarine fissure (Fig. 3).

The distributions of lesions in the white matter corresponded accurately with the macroscopic appearances. The central white matter of the occipital lobes was completely devoid both of myelin sheaths and of axons and consisted of rows of lacunae separated by glial septa of variable thickness (Figs. 4 and 5). Some of the septa separating the lacunae of the same rows were very slender and consisted of a blood vessel and a few glial fibres (Fig. 6). Others formed somewhat wider bridges of tissue, containing blood vessels, proliferated astrocytes, glial fibres, and phagocytic microglia. Similar appearances were seen in the broader septa separating the rows of lacunae from each other. This central area of lacunar rarefaction was surrounded by a zone of demyelination with partial preservation of axons, extending deeply into the subcortical white matter and encroaching in places upon the cortex as noted above. While axons were present in these demyelinated zones their density was less than normal and decreased rapidly towards the central area of cavitation. Astrocytic proliferation and fibrillary gliosis were prominent and phagocytic microglia abundant. Many blood vessels were surrounded by thick cuffs of mononuclear cells, predominantly of microglial origin. Similar appearances were observed in the splenium of the corpus callosum and the adjacent white matter of the cingulate gyrus.

The deep structures of the hemispheres were normal with the exception of the lateral geniculate bodies, which

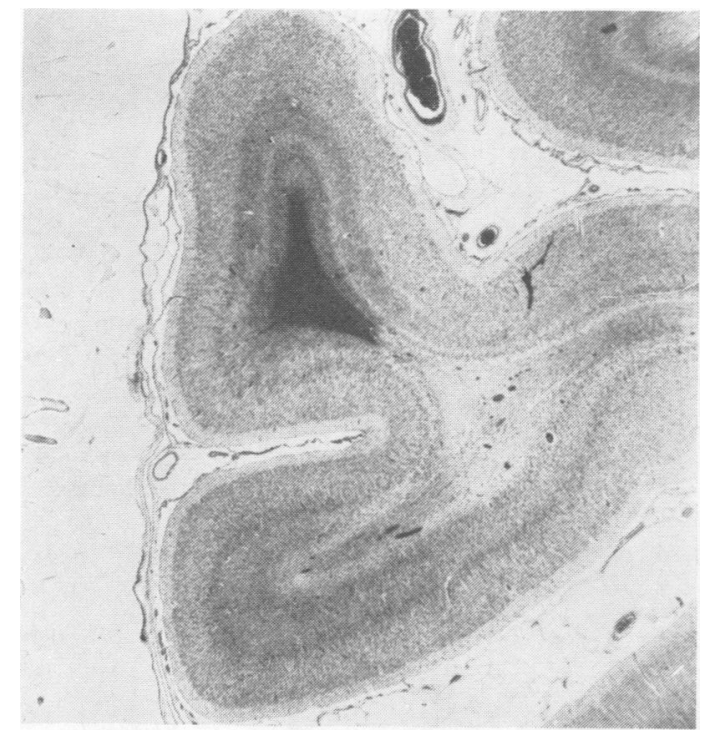

FIG. 2. Right visual cortex: myelin is preserved only in the tips of the gyri while the deeper parts are totally demyelinated. Klüver and Barrera's luxol fast blue and cresyl violet, $\times 7$. showed severe atrophy with almost total loss of neurones.

The optic pathway showed patchy demyelination in the optic nerves and chiasm. The intra-orbital part of the right optic nerve showed total loss of myelin and severe, but not complete, loss of axons (Fig. 7). The nerve appeared very cellular owing to astrocytic and microglial proliferation. A dense network of fine glial fibrils was present throughout the nerve. The left optic nerve appeared slender, the cords of myelinated fibres were thinner than normal and the fibrous septa crowded together (Fig. 8). Despite this appearance of mild atrophy the density of axons and their myelination seemed normal and fibrillary gliosis minimal. The chiasm showed massive demyelination of the decussation in continuation with the plaque in the right optic nerve (Fig. 9). There was also a small irregular plaque at the left lateral margin of the chiasm.

In the spinal cord the lesions became apparent at the level C4 where a central area of recent necrosis with breakdown of axons and myelin sheaths occupied mainly the deeper parts of the posterior columns (Fig. 10). Caudally, the lesion increased rapidly in size and at C8 occupied almost the entire cross-section of the cord with the exception of a thin peripheral rim of preserved myelinated fibres (Fig. 11). Amid this massive breakdown of the white matter the grey matter appeared relatively well preserved and the neurones of the anterior and posterior horns were apparently unaffected. The lesion reached its maximum intensity at $\mathrm{T} 4$ where destruction of the cord was total and apparently of longer duration than in the cervical segments (Fig. 12). There was diffuse proliferation of phagocytic microglia and patchy infiltration by polymorphonuclear leucocytes, but no

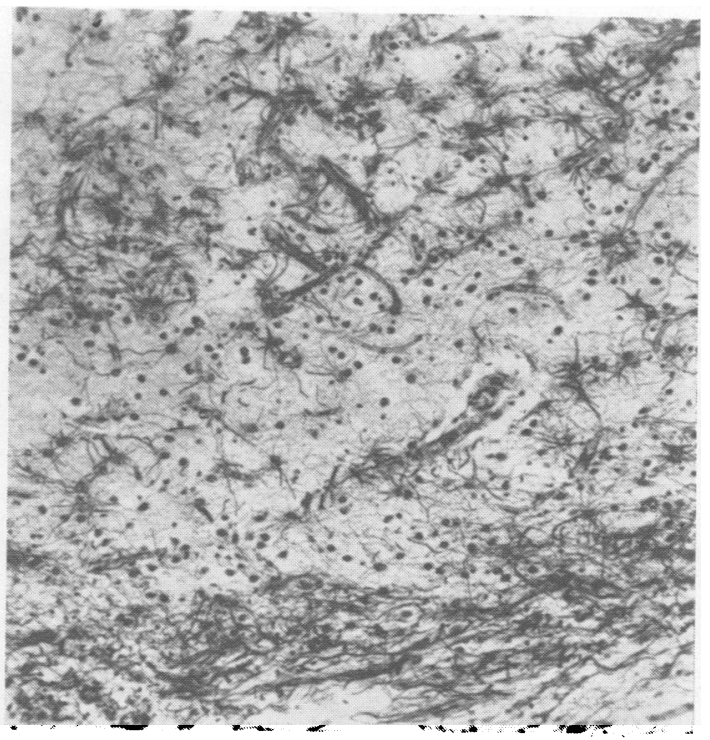

FIG. 3. Right occipital cortex: proliferation of spider astrocytes in floor of calcarine fissure adjacent to glial wall of medial row of lacunae. Holzer's method for glial fibres, $\times 150$. 


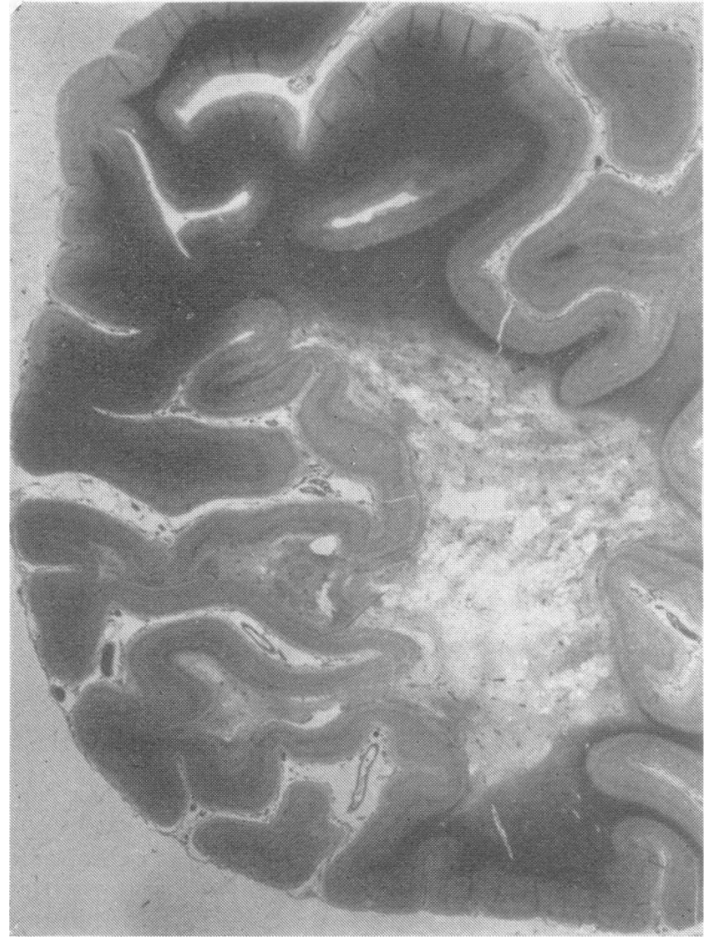

FIG. 4. Section of right occipital lobe stained for myelin showing extent of demyelination and lacunar rarefaction. Klüver-Barrera, $\times 1 \cdot 8$.

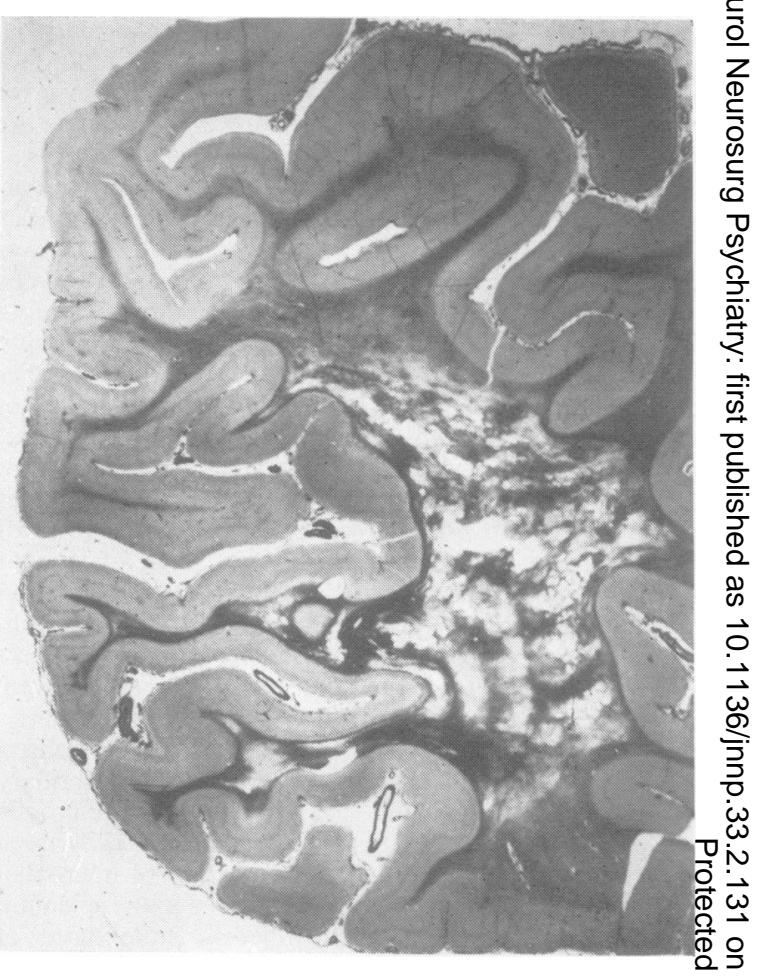

FIG. 5. Adjacent section stained for glial fibres: the concentric arrangement of glial septa is clearly seen $\frac{7}{0}$ Holzer, $\times 1 \cdot 8$.

FIG. 6. Right occipital lobe: groups of thinwalled lacunae separated by thick glial septum.

Holzer, $\times 45$.

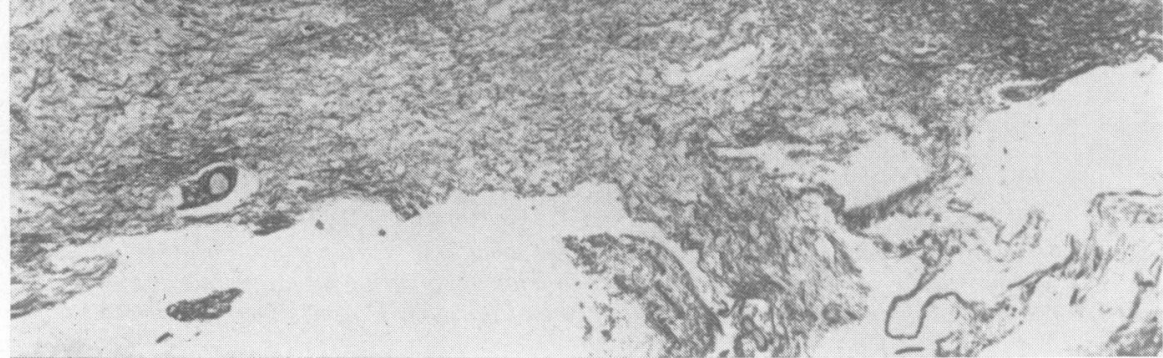




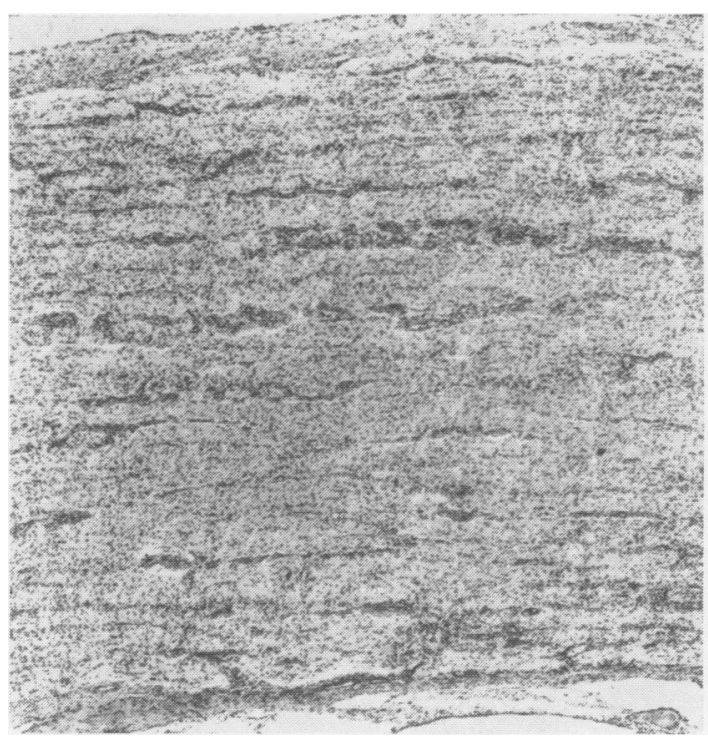

FIG. 7. Right optic nerve: total loss of myelin and increased cellularity due to proliferation of glial cells. Klüver-Barrera, $\times 30$.

astrocytic reaction. Similar appearances were seen in lower segments of the thoracic cord down to T10, but with gradual reappearance of small islands of myelinated fibres. At T12 the necrosis was reduced to a central core involving the base of the posterior columns. This continued as a thin tail of circumscribed necrosis in the left posterior column down to the level L5 (Fig. 13).

\section{DISCUSSION}

The clinical diagnosis in this case presented considerable difficulty. In retrospect, when the full records were brought together from the five hospitals in which the patient had been investigated, the evolution of the disease and the distribution of the lesions might have suggested a demyelinating disease, possibly neuromyelitis optica. The unusually

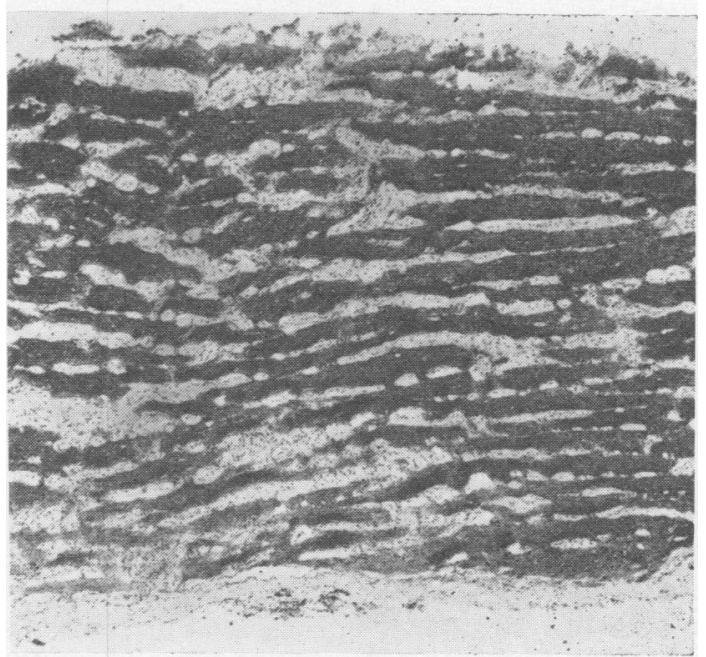

FIG. 8. Left optic nerve: preservation of slender cords of myelinated fibres. Klüver-Barrera, $\times 30$.

advanced age should not have precluded the diagnosis, as the incidence of this disease covers a span from 5 to over 60 years (McAlpine, 1938).

The clinicopathological concept of neuropticomyelitis is not entirely clear. The clinical picture was fully reviewed by Stansbury (1949) on a material consisting of 200 reported cases. The syndrome consists of blindness, temporary or permanent, followed after a variable, though usually short, interval by paraplegia of rapid onset. The disease is progressive and often rapidly fatal, but cases running a remitting and relapsing course have been reported. Pathologically the lesions fall into three groups (Greenfield and Norman, 1963): some are examples of multiple sclerosis involving predominantly the optic pathways and the spinal cord, others resemble

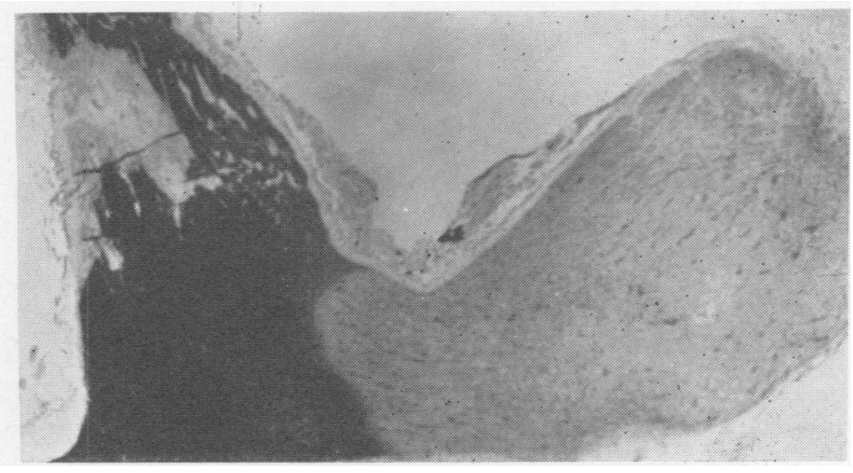

FIG. 9. Optic chiasm: plaque of demyelination extending from right optic nerve into decussation; also small plaque at base of left optic nerve. Klüver-Barrera, $\times 7$. 


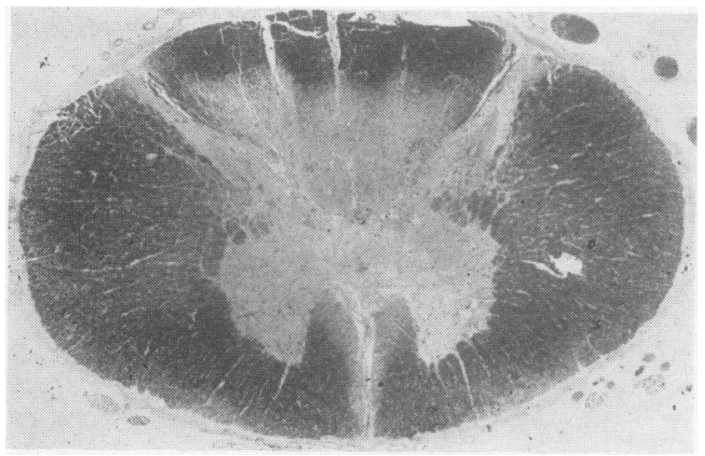

FIG. 10. Spinal cord, segment C4: central area of necrosis, affecting mainly ventral two thirds of posterior columns. Klüver-Barrera, $\times 7$.

acute perivenous encephalomyelitis of similar distribution, others still consist of demyelinating lesions in the optic pathways associated with acute necrotic myelopathy. Hughes (1966) suggested that only the last group formed a separate entity for which the term neuromyelitis optica should be retained.

The association of necrotizing myelopathy, indistinguishable from the isolated myelomalacia described originally by Bassoe and Hassin (1921) and fully reviewed by Hughes (1961), with asymmetrical plaques of demyelination in the optic nerves clearly places our case in this last group. The unusual finding was the lesion affecting both occipital lobes and the splenium of the corpus callosum and closely resembling the concentric lacunar leucoencephalopathy of Grcević (1960). Demyelinating lesions in the occipital lobes have been reported in

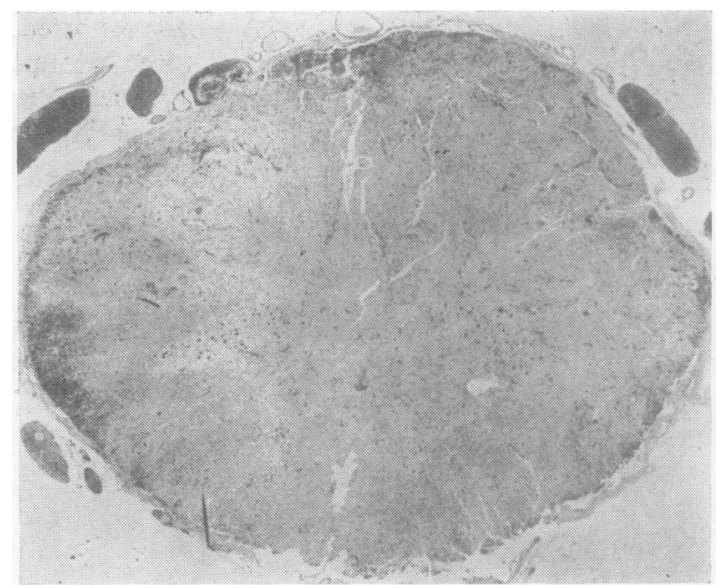

FIG. 11. Spinal cord, segment C8: almost total necrosis with partial preservation of thin peripheral rim of myelin. Klüver-Barrera, $\times 7$.

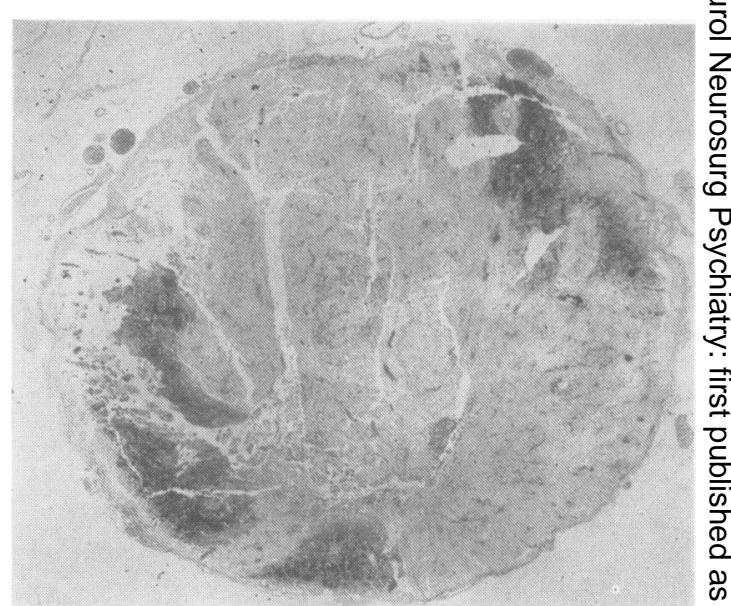

FIG. 12. Spinal cord, segment T4: massive necrosis of $\vec{\circ}$ cord; dark areas represent leucocytic infiltration, not $\overrightarrow{-}$ preservation of myelin. Klüver-Barrera, $\times 7$.

several cases of Devic's disease (Marinesco, Draganesco, Sager, and Grigoresco, 1930; Greenfield, 1950; Paarmann, 1952). The case of Marinesçi et al. is of particular interest in that the extensi⿺辶, $\vec{\omega}$ though strictly unilateral, parieto-occipital lesion consisted of an outer demyelinated and inner lacunar을 zone. The lacunae, however, were not arrangedconcentrically, and the intervening tissue contained demyelinated axons as well as glial fibres. The significance of the concentric pattern and its possibe $\vec{c}$ relationship to the 'encephalitis periaxialis comcentrica' of Baló (1928) was fully discussed by Grcević and our case throws no new light on this

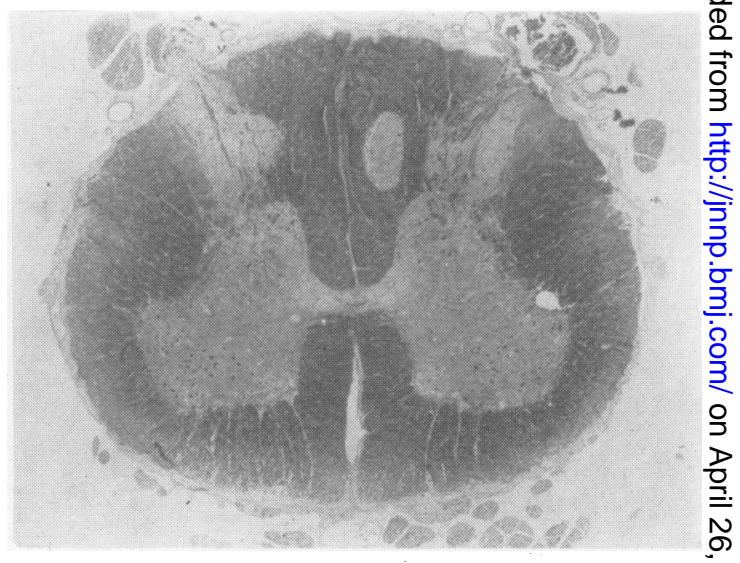

FIG. 13. Spinal cord, segment L5: small circumscribed area of necrosis in one posterior column. Klüver-Barrera, N̦ $\times 7$.

\section{(2)}


problem. The essential difference between lacunar encephalopathy and Baló's sclerosis lies in the fact that in the latter zones of demyelination are separated by lamellae of preserved white matter, in the lacunar type zones of cavitation alternate with glial septa devoid of axons and myelin sheaths.

Our case differs from that of Grcević mainly in the distribution of the lesions which in his affected the frontal lobes and the anterior third of the corpus callosum. The optic nerves and spinal cord were not examined. While there was no clinical suggestion of a myelopathy the history of recurrent episodes of loss of vision progressing towards almost total blindness suggested involvement of the optic pathways presumably by a demyelinating process. Nevertheless Grcević considered that the available evidence did not warrant the inclusion of his case in any definite nosological group.

We feel that our case clarifies the nosological position of concentric lacunar leucoencephalopathy. The association with typical neuropticomyelitis places this lesion within the context of demyelinating diseases.

\section{SUMMARY}

A man aged 64 presented with left homonymous hemianopia rapidly progressing to total cortical blindness. This was followed by a transient left hemiparesis and grand mal seizures. Fourteen months later he developed a rapidly progressive ascending myelopathy to which he succumbed. Post-mortem examination of the nervous system revealed plaques of demyelination in the optic nerves and chiasm, an acute necrotizing myelopathy, and bilateral lesions in the occipital white matter identical with those described by Grcevic under the name of concentric lacunar leucoencephalopathy. The association of this condition with typical neuropticomyelitis suggests its close relationship to the demyelinating diseases.

We are indebted for the excellent documentation of this case, and for permission to publish it, to Dr. R. A. Henson and Mr. T. T. King, and also to Dr. F. Lees, Mr. B. Fairburn, and Lt. Col. A. V. Forage.

\section{REFERENCES}

Baló, J. (1928). Encephalitis periaxialis concentrica. Arch. Neurol. Psychiat. (Chic.), 19, 242-264.

Bassoe, P., and Hassin, G. B. (1921). Myelitis and myelomalacia: a clinico-pathologic study with remarks on the fate of gitter cells. Arch. Neurol. Psychiat. (Chic.), 6, 32-43.

Grcević, N. (1960). Concentric lacunar leukoencephalopathy. Arch. Neurol. (Chic.), 2, 266-273.

Greenfield, J. G. (1950). The classification of diffuse demyelinating sclerosis of the brain on the basis of pathogenesis. Folia psychiat. neerl., 53, 255-267.

- and Norman, R. M. (1963). Neuropticomyelit is (neuromyelit is optica, Devic's syndrome), in Greenfield's Neuropathology, pp. 496-499, 2nd edition. Edited by W. Blackwood, W. H. McMenemey, A. Meyer, and D. S. Russell. Arnold: London.

Hughes, J. T. (1961). Acute Idiopathic Myelomalacia. MD Thesis, Victoria University: Manchester.

(1966). Pathology of the Spinal Cord. Pp. 150-152. Lloyd-Luke: London.

McAlpine, D. (1938). Familial neuromyelitis optica: its occurrence in identical twins. Brain, 61, 430-448.

Marinesco, G., Draganesco, S., Sager, O., and Grigoresco, D. (1930). Sur une forme particulière anatomo-clinique d'ophtalmoneuromyélite (Ophtalmo-encéphalo-myélite). Rev. neurol., 3, 193-228.

Paarmann, H. F. (1952). Beitrag zur Neuromyelitis Optica. Dtsch. $Z$. Nervenheilk., 168, 384-400.

Stansbury, F. C. (1949). Neuromyelitis optica (Devic's disease): presentation of 5 cases, with pathologic study, and review of literature. Arch. Ophthal., 42, 292-335, and 465-501. 